\title{
Factors affecting length of stay in teaching hospitals of a middle-income country
}

\author{
Omid Khosravizadeh $^{1}$, Soudabeh Vatankhah ${ }^{2}$, Peivand Bastani ${ }^{3}$, Rohollah Kalhor ${ }^{4}$, Samira Alirezaei ${ }^{5}$, Farzane \\ Doosty $^{6}$
}

${ }^{1}$ Ph.D. Candidate of Health Services Management, Health Management and Economics Research Center, Iran University of Medical Sciences, Tehran, Iran

${ }^{2}$ Associate Professor of Health Services Management Department, School of Health Management and Information Sciences, Iran University of Medical Sciences, Tehran, Iran

${ }^{3}$ Assistant Professor, Health Human Resources Research Center, School of Management and Medical Informatics, Shiraz University of Medical Sciences, Shiraz, Iran

${ }^{4}$ Assistant Professor, Social Determinants of Health Research Center, Qazvin University of Medical Sciences, Qazvin, Iran

${ }^{5}$ Health Services Management Research Center, Institute for Futures Studies in Health, Kerman University of Medical Sciences, Kerman, Iran

${ }^{6}$ Students Research Committee, Hormozgan University of Medical Sciences, Bandar Abbas, Iran

\section{Type of article: Original}

\begin{abstract}
Introduction: The length of stay (LOS) in hospitals is a widely used and important criteria for evaluating hospital performance. The aim of this study was to determine factors affecting LOS in teaching hospitals of Qazvin Providence.

Methods: In this cross-sectional study, patients' health records were randomly selected from archives in teaching hospitals of Qazvin in 2013. Data were collected through a data entry form and were analyzed using Kolmogorov-Smirnov, Kruskal-Wallis, and Mann-Whitney U tests at the significant level of 0.05.

Results: The mean of hospital LOS was $5.45 \pm 6.14$ days. Age, employment, marital status, history of previous admission, patient condition at discharge, method of payment, and type of treatment had an impact on LOS $(\mathrm{p}<0.05)$. Other factors, including gender, place of residence, and type of admission, did not affect LOS.

Conclusion: Because hospitals consume a perceptible part of resources in a health system, controlled and optimized use of its resources help to save a lot. Therefore, this study showed many clinical and nonclinical factors affect LOS in evaluating these factors, which may reduce inappropriate hospital stays and decrease costs.

Keywords: Length of stay, Clinical and nonclinical factors, Hospital performance, Teaching hospital
\end{abstract}

\section{Introduction}

Hospitals are an important part of a health system that, in coordination with other parts, provide health to the community. In fact, hospitals have a key role in providing health services; therefore, they have a major impact on the efficiency of a health system (1). The most important tool for evaluating hospital performance is hospital indicators. Among these indicators, length of stay (LOS) in a hospital is one of the most important and simplest indicators that is widely used today. This indicator is used for different goals, including management of hospital care, quality control, appropriateness of using hospital services, and hospital planning (2). LOS in a hospital is the time interval between admission and discharge from the hospital and evaluates bed occupancy and efficiency of hospital wards (3). With little attention to annual cost of hospital beds without considering unavoidable costs, the amount of ineffective and imposed costs, which are forced into a health system because of inappropriate stay of

\section{Corresponding author:}

Associate Professor Dr. Soudabeh Vatankhah, Health Services Management Department, School of Health Management and Information Sciences, Iran University of Medical Sciences, Tehran, Iran.

Tel: +98.9121986451, Fax: +98.02188883334,Email: vatankhah_s@yahoo.com

Received: March 30, 2016, Accepted: July 24, 2016, Published: October 2016

iThenticate screening: July 23, 2016, English editing: September 02, 2016, Quality control: October 06, 2016

(C) 2016 The Authors. This is an open access article under the terms of the Creative Commons Attribution-NonCommercialNoDerivs License, which permits use and distribution in any medium, provided the original work is properly cited, the use is non-commercial and no modifications or adaptations are made. 
patients, will be determined (4). To reduce hospital costs, different parts of health systems vigorously try to reduce unnecessary LOS of patients (5). However, it must be considered that overstaying of patients or staying less than what is really required affects the cost and quality of provided care. Excessive hospitalization increases use of limited resources and cost, while under-hospitalization causes unsatisfactory outcomes in treatment (6). Thus reducing the inappropriate stay of patients in a hospital not only decreases cost and improves hospital performance but also reduces false bed occupancy rate and increases hospital productivity (7). In this regard, Iranian evidence indicates that the highest rate of ALS belongs to the educational hospitals in rich areas with more than 300 beds (4.33 days); in contrast, those non-educational hospitals with 51-300 beds located in poor areas had the lowest ALS with 1.29 days, which is higher than Iranian Ministry of Health and Medical Education's standards (8). These evidences indicate the importance of LOS and its related determinant factors in an Iranian health system. Research showed that LOS is a variable dependent on provided health services and demands for it. Provided health services consist of hospital beds, method of payment and hospital discharge policy; demand for health services include severity of disease, direct and indirect cost of patients, and patient comorbid diseases (9). Tiessen conducted a study in two countries of Japan and Canada, which evaluated factors affecting LOS showed that different factors, including age, gender, type of intervention, type of disease, and many clinical and nonclinical factors, even culture, cause huge difference in LOS between these two countries (10). The result of Yin study indicated that a significant relation existed between level of hospital financial support dependent on patient activity and LOS in elderly people suffering from ischemic heart disease (11). One study in an Arabic county determined that nationality and race had an impact on LOS in hospitals (12). Finally, many studies have assessed clinical and nonclinical factors that are related to patients' LOS, which determine the importance of this topic (13-15). The present study evaluated some of clinical and nonclinical factors having an impact on LOS in teaching hospitals of Qazvin, with the aim of determining these factors and optimizing LOS, which could improve clinical performance and effective utilization of hospital resources.

\section{Material and Methods}

A cross-sectional study was conducted with the aim of evaluating factors that affect patients' LOS in teaching hospitals of Qazvin in the first six months of 2013. The study population was all the hospitalized patients in teaching centers of Velayat, Bo-Alisina, Ghods, and Shahid Rajaie. At first, each of the selected centers were determined as a class, then 640 study samples, which were taken from health records of hospitalized patients, were randomly selected from each hospital in proportion to their active bed. The required data for descriptive and analytical goals of study were extracted from health records using a data entry form. This form had two parts for registering demographic data such as age, gender, marital status, occupation, and place of residence, along with information related to hospitalization, including history of admission to hospital, type of admission, type of treatment, patient condition at discharge, and type of payment for hospital costs. The data entry form was filled at hospital archive with direct surveillance of the researcher. The hospital admission form in the above centers includes a part asking patients if their health recodes could be used for research purposes with keeping their personal information confidential. The health records of patients who agreed were used in this study; therefore, a separate consent form was not taken for the matter of this study. The study protocol was approved by Regional Committee of Research Ethics at Qazvin University of Medical Sciences. Also in publishing results, the patient's personal information was kept confidential. The data were analyzed using SPSS software version 18 (SPSS Inc. Chicago, Illinois, USA) and Kolmogorov-Smirnov test, but since the dependent variable of LOS did not follow normal distribution, MannWhitney U and Kruskal-Wallis tests were used to analyze data. The significant level was considered at 0.05 .

\section{Results}

More than half of patients were male (56.6\%). The mean of total hospital stay in Qazvin teaching hospitals was 5.45 \pm 6.13 days. Among demographic factors gender $(p=0.737, z=-0.33)$ and residence $(z=-0.571, p=0.568)$ did not affect LOS. The distribution of LOS in Qazvin hospitals based on demographic variables is demonstrated in Table 1. The study results showed that patients with history of previous hospital admission had longer stay (mean LOS = 6.385 days) compared with others (mean LOS $=5.001$ days). In regards to patient condition at discharge, those patients who passed away had the longest LOS (7.738 days) and those who were discharged based on personal wishes had the shortest LOS (3.553 days); therefore, patient condition at discharge has an effect on LOS. The type of admission did not affect LOS; there were no significant difference in LOS between those who had elective or emergency admissions. The method of payment for hospital expenses had an influence on LOS in a way that patients who were under Relief Committee insurance (a charity committee that pays for poor people's health expenses( had the longest stay (8.833 days) and patients with no insurance had the shortest stay (2.769 days), so variable methods of payment influences length of stay. In respect to the type of treatment, the mean of LOS in 
http://www.ephysician.ir

patients who received medical treatment (5.842 days) was longer than those who received surgical intervention (4.868 days), so the variable type of treatment affected LOS. The distribution of LOS based on clinical and nonclinical variables is demonstrated in Table 2.

Table 1. Distribution of LOS in Qazvin hospitals based on demographic variables

\begin{tabular}{|c|c|c|c|c|c|c|}
\hline \multicolumn{2}{|c|}{ Demographic Variables } & $n$ & $\%$ & Mean & SD & $p$-value \\
\hline \multirow[t]{2}{*}{ Gender } & Male & 362 & 56.6 & 5.51 & 6.48 & \multirow[t]{2}{*}{0.737} \\
\hline & Female & 278 & 43.4 & 5.375 & 5.68 & \\
\hline \multirow[t]{2}{*}{ Marital status } & Bachelor & 177 & 27.7 & 3.875 & 4.17 & \multirow[t]{2}{*}{$<0.001$} \\
\hline & Married & 463 & 72.3 & 6.056 & 6.64 & \\
\hline \multirow[t]{4}{*}{ Age group (year) } & $<20$ & 129 & 20.2 & 3.938 & 4.34 & \multirow[t]{4}{*}{$<0.01$} \\
\hline & $21-30$ & 76 & 11.9 & 3.664 & 4.06 & \\
\hline & $31-40$ & 56 & 8.77 & 4.571 & 7.25 & \\
\hline & $>40$ & 377 & 56.09 & 6.465 & 6.62 & \\
\hline \multirow[t]{7}{*}{ Occupation group } & Self employed & 107 & 16.7 & 5.355 & 6.32 & \multirow[t]{7}{*}{$<0.01$} \\
\hline & Employee & 26 & 4.1 & 6.2 & 8.16 & \\
\hline & Worker & 44 & 6.9 & 4.534 & 4.71 & \\
\hline & Student & 66 & 10.3 & 3.553 & 5.53 & \\
\hline & Home maker & 223 & 34.8 & 5.538 & 5.89 & \\
\hline & Unemployed & 79 & 12.3 & 4.607 & 4.84 & \\
\hline & Retired & 94 & 14.7 & 7.670 & 7.79 & \\
\hline \multirow[t]{3}{*}{ Place of residence } & Native & 256 & 40 & 5.858 & 7.02 & \multirow[t]{3}{*}{0.568} \\
\hline & Non-native & 377 & 58.9 & 5.185 & 5.50 & \\
\hline & Unclear & 7 & 1.1 & - & - & \\
\hline
\end{tabular}

Table 2. Distribution of LOS based on clinical and nonclinical variables

\begin{tabular}{|c|c|c|c|c|c|c|}
\hline Variable & Variable Level & $n$ & $\%$ & Mean & SD & $p$-value \\
\hline \multirow[t]{2}{*}{ History of previous admission } & Yes & 209 & 32.7 & 6.385 & 6.155 & \multirow[t]{2}{*}{$<0.001$} \\
\hline & No & 431 & 67.3 & 5.001 & 6.092 & \\
\hline \multirow[t]{5}{*}{ Patient condition at discharge } & Improved & 484 & 75.6 & 5.554 & 5.592 & \multirow[t]{5}{*}{0.002} \\
\hline & Expired & 42 & 6.6 & 7.738 & 9.563 & \\
\hline & Discharge with personal wish & 39 & 6.1 & 2.842 & 1.990 & \\
\hline & Transfer to another health center & 7 & 1.1 & 3.857 & 3.933 & \\
\hline & Unclear & 68 & 10.6 & - & - & \\
\hline \multirow{2}{*}{ Type of admission } & Elective & 234 & 36.6 & 5.536 & 5.994 & \multirow[t]{2}{*}{0.525} \\
\hline & Emergency & 405 & 63.3 & 5.406 & 6.241 & \\
\hline \multirow[t]{7}{*}{ Method of payment } & No insurance & 40 & 6.25 & 2.769 & 3.316 & \multirow[t]{7}{*}{$<0.001$} \\
\hline & Treatment services & 206 & 32.18 & 6.085 & 6.641 & \\
\hline & Social Security & 330 & 51.56 & 4.951 & 4.866 & \\
\hline & Army & 23 & 3.59 & 8.326 & 12.285 & \\
\hline & Relief committee & 18 & 2.81 & 8.833 & 10.798 & \\
\hline & Health Deputy of Ministry of Health & 12 & 1.87 & 6.250 & 6.648 & \\
\hline & Other & 11 & 1.71 & 6 & 4.604 & \\
\hline \multirow[t]{2}{*}{ Type of treatment } & Surgery & 255 & 39.8 & 4.868 & 5.597 & \\
\hline & Medical & 385 & 60.2 & 5.842 & 6.456 & \\
\hline
\end{tabular}

\section{Discussion}

The mean LOS in teaching centers of Qazvin Medical Sciences University was $5.45 \pm 6.14$ days. Among four evaluated hospitals, the Velayat Hospital with mean LOS of 7.57 days had the longest, and Ghods Hospital with mean LOS of 3.76 days had the shortest LOS in hospital. It could be related to the fact that the newly established Velayat Hospital with 160 active beds admits patients in a variety of therapeutic range and different specialties, which could affect LOS. Furthermore, in Ghods Hospital most of surgeries are ENT surgeries, which could be a reason for shorter LOS. Gender did not affect LOS, and the mean LOS was not significantly different between males and females in Qazvin hospitals. This could be explained by the fact that one of the hospitals is only active in 
gynecology and obstetrics, and most of deliveries and female surgeries are performed there. However, the Singh study in India showed that gender has an influence on LOS (16). Among different age groups, patients older than 40 years had the longest stay, and age groups between 20 to 30 years old had the shortest stay, so patient age influences LOS. The reason for increased LOS along with age could be the presence of chronic disease in older ages, which required a longer hospital stay. A Lim study in south Thailand showed that age affected LOS, and with increasing age LOS increases as well (17). The current study results indicated that marital status affects LOS, and married patients had longer stays than bachelors, which is similar to the results of other studies $(18,19)$. Regarding occupation, the retired patients had the longest LOS and students had the shortest. The reason for shorter stays of students could be the existence of acute and early treated diseases among this group. In addition, retired people usually have chronic diseases, which increase their LOS. The results of a previous study in Iran showed that housewives had the shortest LOS (20). The LOS of Qazvin native people was longer than non-natives, while the number of hospitalized non-natives was higher. Because Qazvin's teaching hospitals have good medical facilities and many specialists in different fields, patients from all over the province refer to them for treatment. Studies from other provinces in Iran also had shown that place of residence affect LOS in hospitals $(21,22)$. In respect to the history of previous hospital admission, the study results showed that patients with this history had longer LOS. A study in one of the Tabriz province's hospitals also had shown that history of previous admission influence LOS (23). In regard to patient condition at discharge, patients who eventually passed away in a hospital had the longest LOS. Other studies also confirmed that patient condition at discharge affected LOS $(24,25)$. In the present study in 68 reviewed health records, the patient condition at discharge was unclear: the weakness of the HIS system of studied hospitals in documenting patient condition was the reason. In this study, LOS was not significantly different between patients with elective and emergency admissions, so type of admission did not affect LOS. The reason could be inappropriate use of emergency department and admission of non-emergency patients from an entrance way into some teaching medical centers, which require attention and revision of admitting criteria. Freitas in his study in Portugal found a significant relation between type of admission and LOS (26). Bolden also in his study had reported that type of admission has an influence on LOS (27). In regard to method of payment, the LOS was longer in those who were treated for free compared with those who had to pay. Similar to this result, another study in one of Tehran's hospitals showed that LOS in patients who had no insurance was less than those who paid by insurance (28). Other studies also have confirmed the relationship between method of payment and LOS $(29,30)$. The results of this study showed that the mean LOS was higher among patients with medical treatment compared with those with surgical intervention because general surgeries usually require less hospitalization compared with medical treatments in intensive care departments such as ICU and CCU. The other reason could be performance of global surgeries in studied hospitals, which require more evaluation to assess its effect on LOS. Some other studies also indicated the effect of type of treatment on LOS (31-33). This study had limitations as follows: the restricted population study that limits the results to Qazvin University of Medical Sciences. In this regard, it is recommended to conduct national surveys for comparing the results in different parts of the country. Furthermore, applying qualitative findings through interviews with hospital management experts can help improve the results.

\section{Conclusions}

Results of present study showed that many clinical and nonclinical factors such as age, employment, marital status, history of previous admission, patient condition at discharge, method of payment, and type of treatment can affect LOS. Because health system resources are limited, especially in hospitals, knowing these factors can help reducing inappropriate LOS and optimizing use of resources as well. Further studies are recommended to evaluate factors affecting LOS and providing solutions to reduce inappropriate hospital stay.

\section{Acknowledgments:}

This article is a part of MSc thesis supported by Iran University of Medical Sciences with grant number of 1392 237. Researchers are grateful to thank all the colleagues especially mangers and personnel of studied hospitals for their cooperation and efforts.

\section{Conflict of Interest:}

There is no conflict of interest to be declared.

Authors' contributions:

All authors contributed to this project and article equally. All authors read and approved the final manuscript. 


\section{References:}

1) Asefzadeh S. Hospital Management and Research. Qazvin: Qazvin University of medical siences. 2010.

2) Atienza N, García-Heras J, Muñoz-Pichardo JM, Villa R. An application of mixture distributions in modelization of length of hospital stay. Stat Med. 2008; 27(9): 1403-20. doi: 10.1002/sim.3029. PMID: 17680551.

3) Ravangard R, Arab M, Zeraati H, Rashidian A, Akbarisari A, Mostaan F. Patients' length of stay in women hospital and its associated clinical and non-clinical factors, tehran, iran. Iran Red Crescent Med J. 2011; 13(5): 309-15. PMID: 22737486, PMCID: PMC3371979.

4) Schoen C, Osborn R, How SK, Doty MM, Peugh J. In chronic condition: experiences of patients with complex health care needs, in eight countries, 2008. Health Aff (Millwood). 2009; 28(1): 1-16. doi: 10.1377/hlthaff.28.1.w1. PMID: 19008253.

5) Siskou O, Kaitelidou D, Economou C, Kostagiolas P, Liaropoulos L. Private expenditure and the role of private health insurance in Greece: status quo and future trends. Eur J Health Econ. 2009; 10(4): 467-74. doi: 10.1007/s10198-009-0164-3. PMID: 19593628.

6) Mawajdeh S, Hayajneh Y, Al-Qutob R. The effect of type of hospital and health insurance on hospital length of stay in Irbid, North Jordan. Health Policy Plan. 1997; 12(2): 166-72. PMID: 10168199.

7) Haqgoshayi E, Narimani M. Day hospital is a model to reduce the length of stay in hospital patients. Health Management. 2012; 14(46): 21-30.

8) Bastani P, Vatankhah S, Salehi M. Performance ratio analysis: a national study on Iranian hospitals affiliated to Ministry of Health and Medical Education. Iranian J Publ Health. 2013; 42(8): 876-82. PMID: 26056642, PMCID: PMC4441919.

9) Clarke A. Why are we trying to reduce length of stay? Evaluation of the costs and benefits of reducing time in hospital must start from the objectives that govern change. Qual Health Care. 1996; 5(3): 172-9. PMID: 10161532, PMCID: PMC1055402.

10) Tiessen J, Kambara H, Sakai T, Kato K, Yamauchi K, McMillan C. What causes international variations in length of stay: A comparative analysis for two inpatient conditions in Japanese and Canadian hospitals. Health Serv Manage Res. 2013; 26(2-3): 86-94. doi: 10.1177/0951484813512287. PMID: 25595005.

11) Yin J, Lurås H, Hagen TP, Dahl FA. The effect of activity-based financing on hospital length of stay for elderly patients suffering from heart diseases in Norway. BMC Health Serv Res. 2013; 13(1): 172. doi: 10.1186/1472-6963-13-172. PMID: 23651910, PMCID: PMC3651263.

12) Al-Jadid MS, Robert AA. Determinants of length of stay in an inpatient stroke rehabilitation unit in Saudi Arabia. Saudi Med J. 2010; 31(2): 189-92. PMID: 20174737.

13) Huang PY, Lin MZ, Wen JP, Li XJ, Shi XL, Zhang HJ, et al. Correlation of early postoperative blood glucose levels with postoperative complications, hospital costs, and length of hospital stay in patients with gastrointestinal malignancies. Endocrine. 2015; 48(1): 187-94. doi: 10.1007/s12020-014-0291-1. PMID: 24853883.

14) Nussbaum DP, Penne K, Stinnett SS, Speicher PJ, Cocieru A, Blazer DG 3rd, et al. A standardized care plan is associated with shorter hospital length of stay in patients undergoing pancreaticoduodenectomy. J Surg Res. 2015; 193(1): 237-45. doi: 10.1016/j.jss.2014.06.036. PMID: 25062813.

15) Lee S, O’Neill A, Park J, Scully L, Shenassa E. Health Insurance Moderates the Association Between Immigrant Length of Stay and Health Status. J Immigr Minor Health. 2012; 14(2): 345-9. PMID: 22512006.

16) Singh $\mathrm{CH}$, Ladusingh L. Inpatient length of stay: a finite mixture modeling analysis. Eur J Health Econ. 2010; 11(2): 119-26. doi: 10.1007/s10198-009-0153-6. PMID: 19430985.

17) Lim A, Tongkumchum $P$. Methods for analyzing hospital length of stay with application to inpatients dying in Southern Thailand. Global Journal of Health Science. 2009; 1(1): 27. doi: 10.5539/gjhs.v1n1p27.

18) Rafiee M, Mohammad T, Behboodyan J. Comparison of different modeling strategies length of stay of patients hospitalized in teaching hospitals in Shiraz. Tabriz Medical University Journal. 2006; 2(29): $43-51$.

19) Toyabe S, Cao P, Abe T, Uchiyama M, Akazawa K. Impact of Sociocultural factors on Hospital Length of stay in children with nephritic syndrome in Japan. Health Policy. 2006; 76(3): 259-65. doi: 10.1016/j.healthpol.2005.06.008. PMID: 16026888.

20) Arab M, Zarei A, Rahimi A, Rezaiean F, Akbari F. Analysis of factors affecting length of stay in public hospitals in Lorestan Province, Iran. Hakim Research Journal. 2010; 12(4): 27-32.

21) Naghavi M, jamshidi H. Utilization of Health services in Iran. Tehran: Ministry of Health press. 2003. 
22) Salesi M. Study of patient's length of stay and its associated factors in Tehran Shariati Hospital's surgical units using Multiple Cox Proportional Hazards Model Tehran. Tehran: Tehran University of Medical Sciences. 2005.

23) Gholivahidi R, Kooshavar H, khodayari R. The Study of patients length of stay and its Associated factors in Tabriz Shahid Madani Cardiovascular Hospital. Journal of Health Administration. 2006; 9(25): 63-9.

24) Sepehri A, Simpson W, Sarma S. The influence of health insurance on hospital admission and length of stay-The case of Vietnam. Soc Sci Med. 2006; 63(7): 1757-70. doi: 10.1016/j.socscimed.2006.04.029. PMID: 16766108.

25) Nietert PJ, Silverstein MD, Silver RM. Hospital admissions, length of stay, charges, and in-hospital death among patients with systemic sclerosis. J Rheumatol. 2001; 28(9): 2031-7. PMID: 11550971.

26) Freitas A, Silva-Costa T, Lopes F, Garcia-Lema I, Teixeira-Pinto A, Brazdil P, et al. Factors influencing hospital high length of stay outliers. BMC Health Serv Res. 2012; 12(1): 265. doi: 10.1186/1472-6963-12265. PMID: 22906386, PMCID: PMC3470984.

27) Bolden L, Wicks MN. Length of stay, admission types, psychiatric diagnoses, and the implications of stigma in African Americans in the nationwide inpatient sample. Issues in Mental Health Nursing. 2005; 26(10): 1043-59. doi: 10.1080/01612840500280703. PMID: 16283998.

28) Gohari M, Vahabi N, Moghadamifard Z. Semi-parametric Cox regression for factors affecting hospitalization length. Daneshvar Pezeshki. 2012; 19(99): 23-30.

29) Brasel KJ, Lim HJ, Nirula R, Weigelt JA. Length of stay: an appropriate quality measure? Archives of Surgery. 2007; 142(5): 461-5. doi: 10.1001/archsurg.142.5.461. PMID: 17515488.

30) Mainous A, Diaz V, Everett C, Knoll M. Impact of insurance and hospital ownership on hospital length of stay among patients with ambulatory care-sensitive conditions. Ann Fam Med. 2011; 9(6): 489-95. doi: 10.1370/afm.1315. PMID: 22084259, PMCID: PMC3252189.

31) Liu Y, Phillips M, Codde J. Factors influencing patients' length of stay. Aust Health Rev. 2001; 24(2): 6370. doi: 10.1071/AH010063. PMID: 11496473.

32) Teke K, Kisa A, Demir C, Ersoy K. Appropriateness of admission and length of stay in a Turkish Military Hospital. J Med Syst. 2004; 28(6): 653-63. PMID: 15615293.

33) Jiménez R, López L, Dominguez D, Fariñas H. Difference between observed and predicted length of stay as an indicator of inpatient care inefficiency. International Journal for Quality in Health Care. 1999; 11(5): 375-84. PMID: 10561028. 